\title{
Author Correction: Stronger zonal convective clustering associated with a wider tropical rain belt
}

\author{
Max Popp \& Sandrine Bony
}

Correction to: Nature Communications https://doi.org/10.1038/s41467-019-12167-9, published online 19 September 2019.

The original version of this Article contained errors in Fig. 1. In Fig. 1a, the label at the top of the left panel incorrectly read ' $S_{\lambda}(P)=$ $1.45, W_{P}=1.08$ and $W_{\omega}=32.3$ '. The correct version reads ' $S_{\lambda}(P)=0.90, W_{P}=0.71$ and $W_{\omega}=25.3$ '. Similarly in Fig. $1 \mathrm{~b}$, the label at the top of the left panel incorrectly read ' $S_{\lambda}(P)=0.90, W_{P}=0.71$ and $W_{\omega}=25.3$ '. The correct version $\operatorname{reads}$ ' $S_{\lambda}(P)=1.45, W_{P}=1.08$ and $W_{\omega}=32.3$ '. This has been corrected in both the PDF and HTML versions of the Article.

Published online: 04 December 2019

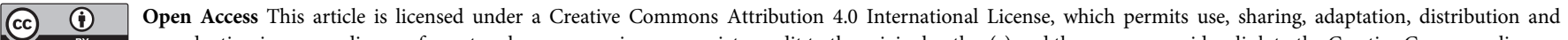

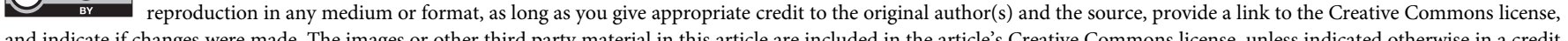

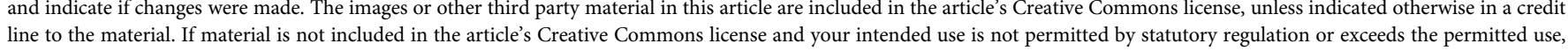
you will need to obtain permission directly from the copyright holder. To view a copy of this license, visit http://creativecommons.org/licenses/by/4.0/.

(c) The Author(s) 2019 